\title{
Locating diametral points
}

\author{
Jin-ichi Itoh Costin Vîlcu Liping Yuan \\ Tudor Zamfirescu
}

October 28, 2019

\begin{abstract}
Let $K$ be a convex body in $\mathbb{R}^{d}$, with $d=2,3$. We determine sharp sufficient conditions for a set $E$ composed of 1,2 , or 3 points of $b d K$, to contain at least one endpoint of a diameter of $K$ (for $d=2,3$ ). We extend this also to convex surfaces, with their intrinsic metric. Our conditions are upper bounds on the sum of the complete angles at the points in $E$. We also show that such criteria do not exist for $n \geq 4$ points.
\end{abstract}

AMS Math. Subj. Classification (2010): 52A10, 52A15, 53C45.

Key words: convex body, diameter, geodesic diameter, diametral point

\section{Introduction}

The tangent cone at a point $x$ in the boundary bd $K$ of a convex body $K$ can be defined using only neighborhoods of $x$ in $\mathrm{bd} K$. So, one doesn't normally expect to get global information about $K$ from the size of the tangent cones at one, two or three points. Nevertheless, in some cases this is what happens!

A convex body $K$ in $\mathbb{R}^{d}$ is a compact convex set with interior points in $\mathbb{R}^{d}$; we shall consider only the cases $d=2,3$. A convex surface in $\mathbb{R}^{3}$ is the boundary of a convex body in $\mathbb{R}^{3}$.

Let $S$ be a convex surface and $x$ a point in $S$. Consider homothetic dilations of $S$ with the centre at $x$ and coefficients of homothety tending to infinity. The limit surface is called the tangent cone at $x$ (see [1]), and is denoted by $T_{x}$. 
If $K$ is a planar convex body then a tangent cone is an angle, and its measure is the angle-measure.

If $K$ is a convex body in $\mathbb{R}^{3}$ then the tangent cone at $x \in \mathrm{bd} K$ can be unfolded in the plane, producing an angle the measure of which is the complete angle at $x$, denoted by $\theta_{x}$.

Denote by $\rho$ the intrinsic metric on the convex surface $S$ (which is derived from the ambient Euclidean distance).

We shall call diameter each line-segment in $K$, or arc in $S$, of length equal to the extrinsic, respectively intrinsic, maximal distance between pairs of points in $K$ or in $S$.

An endpoint of some (intrinsic or extrinsic) diameter is called an (intrinsic, respectively extrinsic) diametral point.

In this paper, we provide criteria for finding extrinsic diametral points in convex bodies $K \subset \mathbb{R}^{d}, d=2,3$, and criteria for finding intrinsic diametral points in convex surfaces $S=\mathrm{bd} K \subset \mathbb{R}^{3}$. Our criteria consist of upper bounds on the sum of the complete angles at 1,2, or 3 points.

We also show that such criteria do not exist for $n \geq 4$ points.

Related to our results in Section 4 is the following one, obtained by $\mathrm{J}$. Itoh and C. Vîlcu [4]. Each point $y$ in a convex surface $S$ with complete angle $\theta_{y} \leq \pi$ is a farthest point on $S$, i.e., $y$ is at maximal intrinsic distance from some point in $S$.

Passing from planar convex bodies to convex surfaces is not always obvious. For example, while the diameter of a convex polygon $P$ (in the plane, diameter means extrinsic diameter) with $n$ vertices can be computed in time $O(n)$ [8, the intrinsic diameter of a convex polyhedral surface in $\mathbb{R}^{3}$ with $n$ vertices can be computed in time $O\left(n^{8} \log n\right)$ [2].

Also, it is well-known that diameters of convex polygons must join vertices, but this is not always true for geodesic diameters of convex polyhedral surfaces [7].

There is a nice connection between the lengths of extrinsic and intrinsic diameters of a convex surface, considered by several authors, see [6], [5], [10]: for any convex surface $S$, the former is not larger than $\pi / 2$ times the latter, and equals it if and only if $S$ is a surface of revolution of constant width.

Our results provide another connection. The endpoints of extrinsic and intrinsic diameters of convex bodies and surfaces are in general distinct; yet, 
in some cases, they can be found in the same set, see the remarks at the end of the paper.

A pair of points sees a line-segment under the angle $\alpha$ if the sum of the two angles under which they see the line-segment equals $\alpha$.

Let $\sigma$ be an extrinsic diameter of the convex body $K$. A pair of points $u, v \in K \backslash \sigma$ is said to be a $\sigma$-separated pair if the line-segment $u v$ meets $\sigma$ [11].

A segment on the convex surface $S$ is an arc (path) on $S$ realizing the intrinsic distance between its endpoints. If $\sigma$ is an intrinsic diameter, i.e. a longest segment, of $S$, then a pair of points $u, v \in S \backslash \sigma$ is said to be $\sigma$-separated if some segment from $u$ to $v$ meets $\sigma$ [1].

For $M \subset \mathbb{R}^{d}$, we denote by $\bar{M}$ its affine hull, by $\operatorname{int} M$ the relative interior of $M$ (i.e., in the topology of $\bar{M}$ ) and by bd $M$ the relative boundary of $M$.

For distinct $x, y \in \mathbb{R}^{d}$, let $x y$ be the line-segment from $x$ to $y$; thus, $\overline{x y}$ is the line through $x, y$. We put $x_{1} \ldots x_{n}=\operatorname{conv}\left\{x_{1}, \ldots, x_{n}\right\}$.

\section{Planar convex bodies}

Let $K$ be a planar convex body and $x$ a boundary point of $K$.

We denote by $X$ the angle of $\operatorname{bd} K$ at $x$ towards $K$ (so $X \leq \pi$ ), and keep this habit for any boundary point; so, $Y$ is the angle at $y$, and so on.

We shall repeatedly use the next result.

Lemma 2.1 (T. Zamfirescu [11]). For any diameter uv of a planar convex

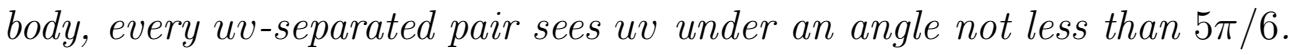

Lemma 2.2. Assume in the convex quadrilateral $Q=x y z w$ we have $X+Y \leq$ $\pi$. Then at least one of the vertices $x, y$ is a diametral point of $Q$.

Proof. Assume $x, y$ are not diametral points of $Q$. Then the side $z w$ is longer than the diagonals $x z$ and $y w$, whence $W<\angle w x z<X$ and $Z<\angle w y z<Y$. It follows that

$$
2 \pi=X+Y+Z+W<2(X+Y) \leq 2 \pi
$$

absurd. 
Theorem 2.3. Let $K$ be a planar convex body.

(i) Any point $x \in \mathrm{bd} K$ with $X \leq \pi / 3$ is a diametral point of $K$. If $K$ has two such points, they determine a diameter of $K$.

(ii) Among any two points $x, y \in \mathrm{bd} K$ with $X+Y \leq 5 \pi / 6$ there exists a diametral point of $K$.

(iii) Among any three points $x, y, z \in \operatorname{bd} K$ with $X+Y+Z \leq 4 \pi / 3$ there exists a diametral point of $K$.

Proof. (i) Assume the existence of a diameter $y z$ of $K$, with $y, z$ different from $x$. It follows that in the triangle $x y z$ the angle at $x$ is not smaller than the other two, whence the triangle is equilateral. Hence, $x y$ and $x z$ are diameters, too. Thus, (i) is proven.

For the rest of the proof (parts (ii) and (iii)), assume the conclusion does not hold, and let $u v$ be a diameter of $K$.

(ii) If $x$ and $y$ are not $u v$-separated, then we have the quadrilateral $x y v u$. By Lemma 2.2, $X+Y \geq \angle u x y+\angle x y v>\pi$, which contradicts our hypothesis.

So $x$ and $y$ are $u v$-separated; then, by Lemma 2.1. $X+Y \geq 5 \pi / 6$. This and the hypothesis imply $X+Y=5 \pi / 6$.

Assume that $K$ is not the quadrilateral xuyv. Then the sum of the angles of $x u y v$ at $x$ and $y$ is less than $5 \pi / 6$, in contradiction with Lemma 2.1, applied to $x u y v$.

So $K$ is the quadrilateral xuyv. Slightly moving $x$ out of $K$ along the line $\overline{x y}$ would provide quadrilaterals $K^{\prime}=x^{\prime} u y v$ with $x^{\prime}, y$ uv-separated and $X^{\prime}+Y<5 \pi / 6$. By Lemma 2.1, $u v$ is no longer a diameter of $K^{\prime}$, so $x^{\prime}$ is a diametral point of $K^{\prime}$. Now, let $x^{\prime}$ converge back towards $x$. Then $K^{\prime} \rightarrow K$, which implies that $x$ is a diametral point of $K$, contradicting our assumption.

(iii) Assume first that $x, y, z$ are all on one side of $\overline{u v}$. Lemma 2.2 gives

$$
X+Y>\pi, \quad X+Z>\pi, \quad Y+Z>\pi,
$$

so $X+Y+Z>3 \pi / 2$, contradicting $X+Y+Z \leq 4 \pi / 3$.

Hence, we can assume that $x, y$ are on one side of $\overline{u v}$ and $z$ on the other side. The previous case (ii) and Lemma 2.2 imply

$$
X+Y>\pi, \quad X+Z>5 \pi / 6, \quad Y+Z>5 \pi / 6 .
$$

Summing up, we get $X+Y+Z>4 \pi / 3$, which contradicts the hypothesis. 
All bounds in Theorem 2.3 are sharp, as one can see from the following examples.

(i) Consider an isosceles triangle $\Delta=x y z$ with equal sides $\|x-y\|=$ $\|x-z\|$ and $X=\pi / 3+\varepsilon$, with $\varepsilon$ arbitrarily small. Clearly, $x$ is not a diametral point of $\Delta$.

(ii) Consider a convex quadrilateral $Q^{\prime}=x^{\prime} u y^{\prime} v$ with $\left\|x^{\prime}-v\right\|=\| x^{\prime}-$ $y^{\prime}\|=\| y^{\prime}-v\|=\| u-v \|$ and $\left\|u-x^{\prime}\right\|=\left\|u-y^{\prime}\right\|$. Then, in $Q^{\prime}, X^{\prime}=Y^{\prime}=$ $U / 2=5 \pi / 12$.

Let $x$ and $y$ be interior points of $Q^{\prime}$, on the line $\overline{x^{\prime} y^{\prime}}$, arbitrarily close to $x$ and $y$, respectively. Then, in $Q=x u y v$, we have $X+Y=5 \pi / 6+\varepsilon$, with $\varepsilon$ arbitrarily small; moreover, $u v$ is the unique diameter of $Q$.

(iii) Consider an equilateral triangle $\Delta=u v z^{\prime}$ and let $m$ be the midpoint of $u v$. Take points $x, y$ on the circle of diameter $u v$, separated from $z^{\prime}$ by $\overline{u v}$, such that $x y \| u v$ and $x$ is arbitrarily close to $u$. Then, in $x u z^{\prime} y, X=Y=$ $\pi / 2+\varepsilon$, with $\varepsilon$ arbitrarily small.

Take a point $z$ on $z^{\prime} m$, such that $\left\|z-z^{\prime}\right\|$ equals the distance between the parallel lines $\overline{x y}$ and $\overline{u v}$. Of course, in xuzvy, $Z=\angle u z v=\pi / 3+\varepsilon^{\prime}$, with $\varepsilon^{\prime}>0$.

Then $X+Y+Z=4 \pi / 3+2 \varepsilon+\varepsilon^{\prime}$, and $2 \varepsilon+\varepsilon^{\prime}$ converges to 0 as $x \rightarrow x^{\prime}$. Moreover, $u v$ is the unique diameter of $x y v z u$.

Corollary 2.4. If the planar convex body $K$, symmetric about $\varnothing$, has a boundary point $x$ with $X \leq 5 \pi / 12$, then $x(-x)$ is a diameter of $K$.

Proof. The sum of the angles at $x$ and $-x$ is not larger than $5 \pi / 6$. Now, by Theorem 2.3 (ii), $x$ or $x^{\prime}$ is a diametral point of $K$. But, by Theorem 4 in [11], the endpoints of each diameter of $K$ are symmetric with respect to $\varnothing$. So, $x(-x)$ is a diameter of $K$.

The above approach cannot be extended to $n \geq 4$ points.

Remark 2.5. There is no non-trivial constant $d(n)$ depending only on $n \geq$ 4, to guarantee that, for any planar convex body $K$, among any $n$ points $x_{1}, \ldots, x_{n}$ in $\mathrm{bd} K$ with $\sum_{i=1}^{n} X_{i} \leq d(n)$ there exists a diametral point of $K$.

Proof. Suppose that such a constant $d(n)$ does exist.

Notice that any points $x_{1}, \ldots, x_{n}$ in the boundary of any planar convex body $K$, form a convex $n$-gon; hence $\sum_{i=1}^{n} X_{i} \geq(n-2) \pi$, and therefore $d(n) \geq(n-2) \pi$. 
Next we show that, for any $\varepsilon>0$, there exist a planar polygon $P$ and $n$ vertices of $P$ with $\sum_{i=1}^{n} X_{i}<(n-2) \pi+\varepsilon$, none of which is a diametral point of $P$. This implies $d(n) \leq(n-2) \pi$, hence necessarily $d(n)=(n-2) \pi$. In this case, $K$ is precisely the convex $n$-gon with vertices $x_{1}, \ldots, x_{n}$ and, trivially, at least two of them are diametral points.

Let $u v$ be a diameter of a circle $C$. Consider $n \geq 4$ points $x_{1}, \ldots, x_{n}$ on $C$, at least two of them on each side of the line $\overline{u v}$, such that no two of them are diametrally opposite. Let $x_{1}, \ldots, x_{k}$ be on one side and $x_{k+1}, \ldots, x_{n}$ on the other side of $\overline{u v}$.

Of course, in the $n$-gon $x_{1} \ldots x_{n}$, we have $\sum_{i=1}^{n} \angle x_{i} x_{i+1} x_{i+2}=(n-2) \pi$, where indices $i$ are taken modulo $n$. Let $P$ denote the $(n+2)$-gon $u v x_{1} \ldots x_{n}$. By taking $x_{1}, x_{n}$ close to $u$, and $x_{k}, x_{k+1}$ close to $v$, we get $\sum_{i=1}^{n} X_{i}<(n-$ 2) $\pi+\varepsilon$, with $\varepsilon$ arbitrarily small. But $P$ has the unique diameter $u v$.

\section{Convex bodies in $\mathbb{R}^{3}$}

We obtain here results similar to Theorem 2.3, locating diametral points of convex bodies in $\mathbb{R}^{3}$.

We denote by $\theta_{x}$ the complete angle at the point $x$ in $\mathrm{bd} K$, and by $\omega_{x}$ the curvature at $x$; hence, $\omega_{x}=2 \pi-\theta_{x}$.

Theorem 3.1. Let $K$ be a convex body in $\mathbb{R}^{3}$.

(i) Any point $x \in \mathrm{bd} K$ with $\theta_{x} \leq 2 \pi / 3$ is a diametral point of $K$. If $K$ has two such points, they determine a diameter of $K$.

(ii) Among any two points $x, y \in \mathrm{bd} K$ with $\theta_{x}+\theta_{y} \leq 3 \pi / 2$ there exists a diametral point of $K$.

(iii) Among any three points $x, y, z \in \mathrm{bd} K$ with $\theta_{x}+\theta_{y}+\theta_{z} \leq 9 \pi / 4$ there exists a diametral point of $K$.

Proof. ( $i$ ) Assume there exists $K \subset \mathbb{R}^{3}$ and a point $x \in \operatorname{bd} K$ with $\theta_{x} \leq 2 \pi / 3$, which is not diametral.

Let $u v$ be a diameter of $K$. In the planar convex body $K \cap \overline{x u v}$, the angle $X$ at $x$ must be at most $\theta_{x} / 2 \leq \pi / 3$. By Theorem $2.4(i), X>\pi / 3$, and a contradiction is obtained.

(ii) Assume there exists $K \subset \mathbb{R}^{3}$ and points $x, y$ on bd $K$ with $\theta_{x}+\theta_{y} \leq$ $3 \pi / 2$, none of which is a diametral point of $K$. 
Let $u v$ be a diameter of $K$. We consider the (possibly degenerate) tetrahedron $T=u v x y$.

We unfold $x u v \cup$ yuv on a plane, with $x, y$ coming on different sides of $\overline{u v}$. The resulting quadrilateral $Q$ has angles $X, Y, U, V$ at the points corresponding to $x, y, u, v$, respectively. Now, unfold $u x y \cup v x y$ on a plane, with $u, v$ coming on different sides of $\overline{x y}$. The resulting quadrilateral $Q^{\prime}$ has angles $X^{\prime}, Y^{\prime}, U^{\prime}, V^{\prime}$ at the points corresponding to $x, y, u, v$. In $Q^{\prime}$, the length of the diagonal corresponding to $u v$ equals at least $\|u-v\|>\|x-y\|$. By Theorem 2.4 (ii), $X^{\prime}+Y^{\prime}>5 \pi / 6$, whence $U^{\prime}+V^{\prime}<2 \pi-(5 \pi / 6)=7 \pi / 6$.

We have

$$
X+X^{\prime}+Y+Y^{\prime}+U+U^{\prime}+V+V^{\prime}=4 \pi
$$

in $\mathrm{bd} T$.

Since $X+X^{\prime}+Y+Y^{\prime} \leq \theta_{x}+\theta_{y} \leq 3 \pi / 2$, we have $U+U^{\prime}+V+V^{\prime} \geq 5 \pi / 2$. This, together with the inequality $U^{\prime}+V^{\prime}<7 \pi / 6$ obtained above, yields $U+V>4 \pi / 3$. This implies $X+Y<2 \pi / 3$. Hence, $X<\pi / 3$ or $Y<\pi / 3$. Thus, $u v$ cannot be a longest side, in $x u v$ or in yuv, and a contradiction is obtained.

(iii) Suppose $\theta_{x}+\theta_{y}+\theta_{z} \leq 9 \pi / 4$, but there is no diametral point among $x, y, z$. Then, by $(i i), \theta_{x}+\theta_{y}>3 \pi / 2, \theta_{y}+\theta_{z}>3 \pi / 2, \theta_{z}+\theta_{x}>3 \pi / 2$. It follows that $2 \theta_{x}+2 \theta_{y}+2 \theta_{z}>9 \pi / 2$, in contradiction with our hypothesis.

Theorem 3.2. If the convex body $K$, symmetric with respect to $\varnothing$, has a boundary point $x$ with $\theta_{x} \leq 5 \pi / 6$, then $x(-x)$ is a diameter of $K$.

Proof. Obviously, $\theta_{x}=\theta_{-x}$. Assume that $x(-x)$ is not a diameter. Then consider a diameter, which, by Theorem 4 in [11], must join diametrally opposite points. Let $y(-y)$ be that diameter. In the parallelogram $x y(-x)(-y)$, the diagonal $y(-y)$ is the unique diameter of it, so $x$ and $-x$ are not diametral points. By Theorem $2.3(i i), \angle y x(-y)+\angle y(-x)(-y)>5 \pi / 6$.

But $\angle y x(-y)+\angle y(-x)(-y) \leq\left(\theta_{x} / 2\right)+\left(\theta_{-x} / 2\right) \leq 5 \pi / 6$, and we got a contradiction. 


\section{Convex surfaces}

In this section we investigate intrinsic diameters on convex surfaces. We obtain results similar to those in Sections 2 and 3. Roughly speaking, as soon as the curvature concentrated at some points is large enough, they become eligible as diametral points.

Lemma 4.1 (The Pizzetti-Alexandrov comparison theorem ([1], p. 132)). The angles of any geodesic triangle in a convex surface are not smaller than the corresponding angles of the Euclidean triangle with the same side-lengths.

Lemma 4.2 below follows from Alexandrov's Konvexitätsbedingung ([1], p. 130).

Lemma 4.2. Consider a convex surface $S$. Let $a b c \subset S$ and $a^{\prime} b^{\prime} c^{\prime} \subset \mathbb{R}^{2}$ be two triangles as in Lemma 4.1. If $d \in b c, d^{\prime} \in b^{\prime} c^{\prime}$ and $\rho(b, d)=\left\|b^{\prime}-d^{\prime}\right\|$, then $\rho(a, d) \geq\left\|a^{\prime}-d^{\prime}\right\|$.

The following statement is well-known. For a thorough introduction to the theory of critical points for distance functions, see [3].

Lemma 4.3. Each endpoint of a diameter on a convex surface is critical with respect to the other. Consequently, each digon determined by two diameters, with no third diameter passing through its interior, has both angles at most $\pi$.

Theorem 4.4. Let $S$ be a convex surface.

(i) Any point $x \in S$ with $\theta_{x} \leq 2 \pi / 3$ is a diametral point of $S$. If $S$ has two such points, they determine a diameter of $S$.

(ii) Among any two points $x, y \in S$ with $\theta_{x}+\theta_{y} \leq 5 \pi / 3$ there exists a diametral point of $S$.

(iii) Among any three points $x, y, z \in S$ with $\theta_{x}+\theta_{y}+\theta_{z} \leq 5 \pi / 2$ there exists a diametral point of $S$.

Proof. ( $i$ ) Assume a point $x$ on $S$ verifies $\theta_{x} \leq 2 \pi / 3$ and is not a diametral point of $S$. Let $y z$ be a diameter of $S$. There are two geodesic triangles with vertices at $x, y, z$ on $S$, at least one of which has an angle not larger than $\pi / 3$ at $x$. A contradiction now follows from the assumption that $x$ is not a diametral point, Lemma 4.1 and Theorem $2.3(i)$.

Assume now that there are $x, y \in S$ with $\theta_{x}, \theta_{y} \leq 2 \pi / 3$, and take $z \in$ $S \backslash\{x, y\}$. Join $x, y$ and $z$ by segments to form two triangles on $S$. At least 
one of them has its angle at $x$ not larger than $\pi / 3$, so $y z$ is not a diameter of $S$ or $x y$ is a diameter, by the preceding argument. Analogously, $x z$ is not a diameter of $S$ or $x y$ is a diameter.

Since this holds for any $z \in S$ and $x, y$ are diametral points of $S, x y$ must be a diameter of $S$.

For the rest of the proof, assume the conclusions are false and let $u v$ be a diameter of $S$.

The segments joining $u$ and $v$ determine on $S$ one or several digons.

(ii) The points $x, y$ are not inside one digon, say $D$, determined by segments from $u$ to $v$. Indeed, by Lemma 4.3, the total curvature of the interior of $D$ is at most $2 \pi$, hence

$$
2 \pi \geq \omega_{x}+\omega_{y}=4 \pi-\left(\theta_{x}+\theta_{y}\right) \geq \frac{7}{3} \pi>2 \pi
$$

absurd.

Therefore, the points $x, y$ are in distinct digons, and so $x$ and $y$ are $u v$ separated, for some diameter $u v$. Let $\{w\}=u v \cap x y$. Consider the points $x^{\prime}, y^{\prime}, u^{\prime}, v^{\prime}, w^{\prime}$ in $\mathbb{R}^{2}$ such that $w^{\prime} \in u^{\prime} v^{\prime}, x^{\prime} y^{\prime} \cap u^{\prime} v^{\prime} \neq \emptyset,\left\|u^{\prime}-v^{\prime}\right\|=\rho(u, v)$, $\left\|u^{\prime}-x^{\prime}\right\|=\rho(u, x),\left\|u^{\prime}-y^{\prime}\right\|=\rho(u, y),\left\|v^{\prime}-x^{\prime}\right\|=\rho(v, x),\left\|v^{\prime}-y^{\prime}\right\|=\rho(v, y)$, $\left\|u^{\prime}-w^{\prime}\right\|=\rho(u, w)$. By Lemma 4.2, $\left\|x^{\prime}-w^{\prime}\right\| \leq \rho(x, w)$ and $\left\|y^{\prime}-w^{\prime}\right\| \leq$ $\rho(y, w)$.

By Lemma 4.1, $\angle u x v \geq \angle u^{\prime} x^{\prime} v^{\prime}$ and $\angle u y v \geq \angle u^{\prime} y^{\prime} v^{\prime}$.

But

$\left\|x^{\prime}-y^{\prime}\right\| \leq\left\|x^{\prime}-w^{\prime}\right\|+\left\|w^{\prime}-y^{\prime}\right\| \leq \rho(x, w)+\rho(w, y)=\rho(x, y) \leq \rho(u, v)=\left\|u^{\prime}-v^{\prime}\right\|$.

By Theorem 2.3 (ii),

$$
\angle u x v+\angle u y v \geq \angle u^{\prime} x^{\prime} v^{\prime}+\angle u^{\prime} y^{\prime} v^{\prime}>5 \pi / 6 .
$$

But

$$
\angle u x v+\angle u y v \leq\left(\theta_{x} / 2\right)+\left(\theta_{y} / 2\right) \leq 5 \pi / 6,
$$

and a contradiction is obtained.

(iii) Notice that the points $x, y, z$ cannot be all in the same digon determined by segments from $u$ to $v$. Indeed, for three points $x, y, z$ in the same digon, we have, by Lemma $4.3, \omega_{x}+\omega_{y}+\omega_{z} \leq 2 \pi$, hence $\theta_{x}+\theta_{y}+\theta_{z} \geq 4 \pi$, contradicting the hypothesis. 
Assume first that $x, y$ are in one digon, and $z$ in another one. Then, by (ii), $\theta_{x}+\theta_{z}>5 \pi / 3$ and $\theta_{y}+\theta_{z}>5 \pi / 3$. At (ii) we saw that $\theta_{x}+\theta_{y} \geq 2 \pi$. Summing up these inequalities, we get $\theta_{x}+\theta_{y}+\theta_{z}>8 \pi / 3>5 \pi / 2$, impossible.

Hence, $x, y, z$ are in different digons. By $(i i)$, we have $\theta_{x}+\theta_{y}>5 \pi / 3$, $\theta_{y}+\theta_{z}>5 \pi / 3$, and $\theta_{x}+\theta_{z}>5 \pi / 3$, hence $\theta_{x}+\theta_{y}+\theta_{z}>5 \pi / 2$, in contradiction with the hypothesis.

Corollary 4.5. If the convex surface $S$, symmetric about $\varnothing$, has a point $x$ with $\theta_{x} \leq 5 \pi / 6$, then there exists a diameter of $S$ from $x$ to $-x$.

Proof. Since $\theta_{x}=\theta_{-x}$, we have $\theta_{x}+\theta_{-x} \leq 5 \pi / 3$. Now, Theorem 4.4 (ii) implies that $x$ or $x^{\prime}$ is a diametral point of $S$. By Proposition 6 in [9], each diameter of $S$ is realized between diametrally opposite points.

The endpoints of extrinsic and intrinsic diameters of convex bodies or surfaces are in general distinct.

The hypotheses of Corollaries 3.2 and 4.5, are the same. Also, those of Theorem 4.4 and Theorem 3.1 might be simultaneously verified. In these cases, endpoints of both extrinsic and intrinsic diameters of $S=\operatorname{bd} K$ can be found in the same subset of $S$ composed by 1, 2, or 3 points.

Conjecture. In Theorem 3.1 (ii), the inequality $\theta_{x}+\theta_{y} \leq 5 \pi / 3$ suffices to guarantee the existence of a diametral point in $\{x, y\}$.

Open questions. Are the bounds $9 \pi / 4$ in Theorem 3.1 (iii) and $5 \pi / 2$ in Theorem 4.4 (iii) optimal?

\section{Acknowledgements.}

The first author was partially supported by a Grant-in-Aid for Scientific Research (C) (No. 17K05222), Japan Society for Promotion of Science. The last two authors gratefully acknowledge financial support by NSF of China (11871192, 11471095). The last three authors direct their thanks to the Program for Foreign Experts of Hebei Province (No. 2019YX002A). The research of the last author was also partly supported by the International Network GDRI ECO-Math. 


\section{References}

[1] A.D. Alexandrov, Die innere Geometrie der konvexen Flächen, Akademie-Verlag, Berlin, 1955

[2] P. K. Agarwal, B. Aronov, J. O'Rourke and C. A. Schevon, Star unfolding of a polytope with applications, SIAM J. Comput. 26 (1997), $1689-1713$

[3] K. Grove, Critical point theory for distance functions, Amer. Math. Soc. Proc. of Symposia in Pure Mathematics, 54 (1993), 357-385

[4] J. Itoh and C. Vîlcu, Criteria for farthest points on convex surfaces, Math. Nachr. 282 (2009), 1537-1547

[5] E. Makai, Jr., On the geodesic diameter of convex surfaces, Period. Math. Hungar. 4 (1972), 157-161

[6] N. P. Makuha, A connection between the inner and the outer diameters of a general closed convex surface (in Russian), Ukrain. Geometr. Sb. Vyp. 2 (1966), 49-51

[7] J. O'Rourke and C. A. Schevon, Computing the geodesic diameter of a 3-polytope, Proc. 5th ACM Symp. Comput. Geom. (1989), 370-379

[8] M.I. Shamos, Computational geometry, Ph.D. thesis, Yale University, 1978

[9] C. Vîlcu, On two conjectures of Steinhaus, Geom. Dedicata 79 (2000), 267-275

[10] V. A. Zalgaller, The geodesic diameter of a body of constant width, $J$. Math. Sci. (N.Y.) 161 (2009), 373-374

[11] T. Zamfirescu, Viewing and realizing diameters, J. Geom. 88 (2008), 194-199

Jin-ichi Itoh

School of Education, Sugiyama Jogakuen University

17-3 Hoshigaoka-motomachi, Chikusa-ku, Nagoya, 464-8662 Japan

j-itoh@sugiyama-u.ac.jp 


\section{Costin Vîlcu}

Simion Stoilow Institute of Mathematics of the Roumanian Academy

P.O. Box 1-764, 014700 Bucharest, Roumania

Costin.Vilcu@imar.ro

\section{Liping Yuan}

College of Mathematics and Information Science,

Hebei Key Laboratory of Computational Mathematics and Applications,

Hebei Normal University,

050024 Shijiazhuang, P.R. China.

lpyuan@hebtu.edu.cn

Tudor Zamfirescu

Fachbereich Mathematik, Universität Dortmund, 44221 Dortmund, Germany, and

Roumanian Academy, Bucharest, and

College of Mathematics and Information Science,

Hebei Normal University, 050024 Shijiazhuang, P.R. China.

tudor.zamfirescu@mathematik.tu-dortmund.de 